\title{
O Protagonismo do Enfermeiro na Política Nacional de Humanização do Parto e do Nascimento e com vistas a Erradicação de Abuso e Violência Obstétrica
}

\author{
Fabiana Laranjeira Silva ${ }^{1}$; Ana Livia Siqueira Souza ${ }^{2}$; Claudia Daniele Barros Leite-Salgueiro ${ }^{3}$;
} Luciclaudio da Silva Barbosa ${ }^{4}$; Luzineide Lobato ${ }^{5}$; Juliane da Silva Pereira ${ }^{6}$

\begin{abstract}
Resumo: Este estudo teve como objetivo analisar na literatura, as ações de Assistência e Promoção à Saúde da Mulher praticadas pela enfermagem, com ênfase na fase reprodutiva, nos períodos do pré-natal, do parto e do puerpério, e considerando-se a vivência de abuso e violência obstétrica. Para tanto, fora utilizada a metodologia de revisão integrativa da literatura, tendo sido utilizados para análise e segundo critérios de inclusão, 11 artigos publicados nas bases SciELO, LILACS e MEDLINE, publicados entre os anos de 2008 e 2017. Na análise dos resultados obtidos, os artigos analisados discorrem sobre a Política Nacional de Humanização do Parto e do Nascimento (PNHPN), os direitos da mulher nas fases reprodutivas e vivências considerando a complexidade temática. Conclui-se que a vulnerabilidade biopsicossocial das mulheres é potencializada principalmente nas fases do pré-natal e do parto, maximizando os déficits de ações em saúde, principalmente no tocante à humanização, destacando a atuação do enfermeiro para a garantia dos direitos dessa população específica.
\end{abstract}

Palavras-chaves: assistência de enfermagem; cuidado; psicologia; violência obstétrica.

\section{The Protagonism of the Nurse in the National Policy of Humanization of birth with a view to the Eradication of Obstetric Abuse and Violence}

\begin{abstract}
This study aimed to analyze in the literature the actions of Assistance and Promotion to Women's Health practiced by nursing, with emphasis on the reproductive phase, in the prenatal, delivery and puerperium periods, and considering the experience of abuse and obstetric violence. For this purpose, 11 articles published in the SciELO, LILACS and MEDLINE databases published between 2008 and 2017 were used for analysis and according to inclusion criteria. In the analysis of the results obtained, the analyzed articles discuss the National Policy of Humanization of Childbirth and Birth (PNHPN), the rights of women in the reproductive phases and experiences considering the thematic complexity. It is concluded that the biopsychosocial vulnerability of women is potentialized mainly in the prenatal and childbirth phases, maximizing the deficits of health actions, mainly in relation to humanization, emphasizing nursing assistance in the action to guarantee the rights of this specific population.
\end{abstract}

Key-words: nursing care; care; psychology; obstetric violence.

\footnotetext{
${ }^{1}$ Discente do Bacharelado em Enfermagem do Instituto Federal de Educação, Ciência e Tecnologia (IFPE), Campus Pesqueira. Discente de Serviço Social da Universidade Pitágoras UNOPAR. Contato: fabilaranjeira22@ gmail.com .

2 Discente do Bacharelado em Enfermagem do Instituto Federal de Educação, Ciência e Tecnologia (IFPE), Campus Pesqueira. Contato: liviloresouza@gmail.com

${ }^{3}$ Doutora em Psicologia Clínica pela Universidade Católica de Pernambuco (UNICAP), Mestra em Ciências da Saúde pela Universidade de Pernambuco (UPE), Pós Graduada (Lato Sensu) em Saúde Coletiva pela mesma Universidade. Psicóloga formada pela Universidade Católica de Pernambuco (UNICAP). Docente do curso Bacharelado em Enfermagem do Instituto Federal de Educação, Ciência e Tecnologia de Pernambuco (IFPE) - Campus Pesqueira. Contato: claudia.leite@ pesqueira.ifpe.edu.br.

${ }^{4}$ Mestre em Recursos Naturais pela Universidade Federal de Campina Grande (UFCG). Biólogo formado pela Universidade Estadual da Paraíba (UEPB). Docente do Curso de Bacharelado em Enfermagem do Instituto Federal de Educação, Ciência e Tecnologia de Pernambuco (IFPE) - Campus Pesqueira. Coordenador dos laboratórios de morfofisiologias dos processos vitais em enfermagem do Instituto Federal de Educação, Ciência e Tecnologia de Pernambuco (IFPE)-Campus Pesqueira. Contato: luciclaudio@pesqueira.ifpe.edu.br ;

${ }_{5}^{5}$ Biológa (UFRN), Docente e Pesquisadora do Instituto Federal de Educação, Ciência e Tecnologia de Pernambuco (IFPE) - Campus Pesqueira. Mestra em Psicobiologia pela Universidade Federal do Rio Grande do Norte (UFRN). Recife (PE), Brasil. E-mail: luzineidelobato@ gmail.com ${ }^{6}$ Enfermeira formada pelo Instituto Federal de Educação, Ciência e Tecnologia de Pernambuco (IFPE) - Campus Pesqueira. Assistente de saúde da Prefeitura Municipal de Pesqueira-Pe. Contato: julianedasilvapereira@ gmail.com .
} 


\section{Introdução}

A violência obstétrica (VO) é sucedida pelos profissionais de saúde, e evidenciada por um conjunto de atos que infringem os direitos da mulher e do seu bebê, ocorrendo desde o prénatal, parto e pós-parto (PPP). No que tange às ações exercidas ou não pelos profissionais de saúde para com a mulher, em qualquer instituição, seja ela pública ou privada (GACETA OFICIAL DE LA REPÚBLICA BOLIVARIANA DE VENEZUELA, 2014). Levando à interrupção dos processos fisiológicos normais do corpo através de condutas desumanas, acarretando danos psicológicos e físicos. Tais condutas incluem agressões físicas e verbais, negligência, abuso e afronta quanto à autonomia feminina, prevalecendo a decisão profissional (AZEVEDO, 2015).

Antes de ser exercido qualquer ato que venha a agredir psicologicamente ou fisicamente a mulher, é violado o seu poder de escolha para aquele momento idealizado pela mesma; excluindo-a do seu protagonismo, dando espaço unicamente às decisões médicas, por meios de práticas que favoreçam as peculiaridades do profissional, como a colocação da mulher em posição horizontal, que facilita a realização do parto para ele; a impaciência da espera da evolução do seu processo fisiológico, atuando na antecipação do mesmo, para que possam ser realizados diversos partos em um curto período de tempo (SANFELICE et al., 2014).

As violências psicológicas contemplam atos insensíveis, de rebaixamento, ignorância e preconceito. Já a violência sexual, expõe e desrespeita sua intimidade, com o aparecimento ou não de suas partes intimas, proporcionando a invisibilidade do consentimento da mulher (PARTO DO PRINCÍPIO- MULHERES EM REDE PELA MATERNIDADE ATIVA. DOSSIÊ DA VIOLÊNCIA, 2012). No que tange às violências verbais, pode-se evidenciar por parte dos profissionais, condutas voltadas para o não repasse de informações fidedignas, que induzem à aceitação de procedimentos desnecessários para benefício profissional, como exemplos: pouca dilatação, negação/ausência de acompanhante, sofrimento fetal, presença de mecônio, feto envolto por cordão umbilical, feto grande para a idade gestacional (GIG), gestante sem estrutura corporal para a realização do parto normal; sendo estes os principais acontecimentos que a induzem ao ato cirúrgico (RATTNER, 2009).

No que diz respeito aos maus-tratos físicos, são caracterizados pela realização da restrição ao leito no pré-parto, jejum de comida e água, tricotomia (corte e raspagem dos pelos 
pubianos), toques vaginais frequentes e por diversos profissionais, clister (utilizado para a realização da lavagem intestinal), ocitocina sintética (onde acelera o trabalho de parto), posição horizontal da mulher, rompimento artificial da bolsa, anestesia, episiotomia (corte na região do períneo), manobra de Kristeler (quando a barriga é empurrada para forçar a saída da criança), fórceps (instrumento utilizado para a retirada da criança pelo canal vaginal), e a cesariana, sendo esta última, uma das violências físicas mais frequentes, ocorridas, na maioria das vezes, desnecessariamente (RATTNER, 2009).

Em 1880, os partos em seu processo fisiológico eram realizados com a presença das parteiras nos ambientes domiciliares, considerado um local mais seguro, sendo desnecessário a sua realização fora desse âmbito. Caso houvesse alguma intercorrência, era solicitada a presença do médico para a realização de procedimentos mais complexos.

Por meio do crescimento da assistência obstétrica, os hospitais passaram a disponibilizar leitos a fim de comportar essa demanda (LEISTER E RIESCO, 2013). As primeiras cidades a ofertarem esse tipo de serviço foram: Rio de janeiro em 1884, e São Paulo dez anos após. Devido a essa implementação, por volta dos anos de 1940, os partos passaram a ser hospitalizados e mais frequentes, causando uma grande interferência no processo natural, incluindo novos protagonistas nesse momento e elevando a procura dos serviços de saúde; houve também melhorias das técnicas assépticas, cirúrgicas e diminuição das dores, proporcionando naquele período, uma redução dos riscos hospitalares, favorecendo o aumento dos partos cirúrgicos (SANTOS, MELO E CRUZ, 2015).

Essa transição do parto domiciliar, fisiológico e feminino para o parto médicointervencionista e hospitalar, contribuiu para o aumento do número de cesáreas e laqueaduras; essa modificação configurou a realização dessa prática cirúrgica como segura, eficiente e indolor (KNUPP, MELO E OLIVEIRA, 2008).

Em um estudo, foi possível identificar que o estado de Pernambuco apresentou um percentual de 34,20\% de atos cirúrgicos do tipo cesariana (CAMPO, ALCÂNTARA E GOIS, 2015). De acordo com os dados preliminares do Ministério da Saúde, o índice de parto cesáreo apresentou uma queda de 1,5 pontos percentuais em 2015 (BRASIL, 2015). Conforme a portaria n³06/2016, o índice de cesáreas no país se encontra em 56\% (BRASIL, 2016). No Brasil em 2012, a cada 1.000 nascidos vivos, 912 destes foram de partos cesáreos, ocupando o primeiro lugar, seguido do México com 448, Turquia com 427, Itália com 384 e da Coréia com 352, entre outros (BRASIL, 2013). 
Mesmo possuindo uma alta incidência e sendo a primeira escolha de parto, é um procedimento invasivo e que traz grandes riscos para a mãe e o bebê, a cesariana é indicada em casos extremos quando realmente se torna impossível o nascimento da criança pela via de parto normal. O procedimento cirúrgico é recomendado nos casos de apresentação pélvica e transversa, desproporção céfalo-pélvica, em complicações decorrentes de hemorragias no final do ciclo gravídico e doenças hipertensivas da gestação; respectivamente descolamento prematuro de placenta (DPP) e placenta prévia (PP), hipertensão gestacional, pré-eclâmpsia, eclâmpsia e a síndrome de HELLP; diabetes gestacional, amniorrex precoce e sofrimento fetal (CAMPO, ALCÂNTARA E GOIS, 2015).

Por conta do aumento no índice de cesáreas e a realização sem causas aparentes, assim como a ocorrência de atos agressivos contra o binômio mãe-filho, foi vista a necessidade de desenvolver uma política que visasse à redução dessa problemática. Pensando nisso, o Ministério da Saúde elaborou a Política Nacional de Humanização do Parto e do Nascimento (PNHPN), que valoriza a figura materna, como também o seu poder de decisão e o direito a receber informações (BRASIL, 2014).

Nesse sentido, o presente estudo teve o objetivo de refletir sobre abuso e VO e suas possíveis implicações ao psicológico materno. Além disso, demonstrar a atuação da enfermagem para diminuir e combater as mesmas, no processo fisiológico do ciclo gravídicopuerperal.

\section{Método}

Trata-se de uma revisão integrativa. Esta se refere a um levantamento e estudo de literaturas, acerca de uma pergunta norteadora que envolva diferentes ideologias a seu respeito (BREVIDELL E SERTÓRIO, 2010). Deste modo, prosseguimos com as seguintes etapas, a saber: 1- Identificação do tema e seleção da questão de pesquisa (Definição do problema, formulação de uma pergunta de pesquisa, da estratégia de busca, dos descritores e das bases de dados), 2- Estabelecimento dos critérios de inclusão e exclusão (uso das bases de dados, busca dos estudos com base nos critérios de inclusão e exclusão), 3- Identificação dos estudos préselecionados e selecionados (Leitura do resumo, palavras-chaves e título das publicações, 
organização dos estudos pré-selecionados, identificação dos estudos selecionados), 4categorização dos estudos selecionados (Elaboração e uso da matriz de síntese, categorização e analisar as informações, formação de uma biblioteca individual, análise crítica dos estudos selecionados), 5- Análise e interpretação dos resultados (Discussão dos resultados), 6Apresentação da revisão/síntese do conhecimento (Escrita detalhada sobre a revisão, propostas para estúdios futuros) (GRUPO ANIMA EDUCAÇÃO, 2010).

A pergunta que deu início e conduziu o desenvolvimento do estudo foi: "Como ocorre a assistência de enfermagem nas fases reprodutivas da mulher e considerando a vivência de abuso e violência obstétrica?"

O levantamento dos artigos científicos ocorreu nos meses de abril e maio de 2017, por meio da Biblioteca Virtual em Saúde (BVS), nas bases de dados Scientific Electronic Library Online (SciELO), Sistema de Análise e Recuperação de Literatura Médica Online (MEDLINE) e Literatura Latino-Americana e do Caribe em Ciências da Saúde (LILACS), fazendo uso dos descritores em ciências da saúde (DECs): assistência de enfermagem, cuidado, psicologia e violência obstétrica.

A eleição destas bases de dados ocorreu, mediante consideração do quantitativo de indexação de artigos da área da saúde, por indexar artigos nas temáticas relacionadas à enfermagem e ainda, por serem bases que contemplam estudos primários. Os descritores em saúde foram combinados de diferentes maneiras com o objetivo de expandir a busca pelos estudos. Foram consideradas as variações terminológicas, bem como a consideração de sinônimos, todos foram utilizados para realização de uma busca sensibilizada.

No que se refere aos critérios de inclusão, considerou-se: artigos publicados nos anos de 2008 a 2017, em português, completos, disponíveis para download e que abordam sobre violências obstétricas; quanto aos critérios de exclusão foram definidos os artigos que estivessem fora do período estipulado para a pesquisa, publicados em outros idiomas, em outras bases de dados, incompletos e que não contemplassem a temática pesquisada.

Com relação à etapa quatro da revisão integrativa, empregou-se os descritores booleanos “AND" e "OR". Já no decorrer da fase analítica crítica, foi possível classificar os estudos com base nas suas evidências. Sobre esta fase, é importante destacar que "a prática baseada em evidências (PBE) focaliza, em contrapartida, sistemas de classificação de evidências caracterizados de forma hierárquica, dependendo da abordagem metodológica adotada" (SOUZA, SILVA E CARVALHO, 2010). 
A classificação das evidências pode ser de seis diferentes níveis, como a seguir: Nível 1: evidências resultantes da meta-análise de múltiplos estudos clínicos controlados e randomizados; Nível 2: evidências obtidas em estudos individuais com delineamento experimental; Nível 3: evidências de estudos quase-experimentais; Nível 4: evidências de estudos descritivos (não-experimentais) ou com abordagem qualitativa; Nível 5: evidências provenientes de relatos de caso ou de experiência;- Nível 6: evidências baseadas em opiniões de especialistas (SOUZA, SILVA E CARVALHO, 2010).

Após o processo de leitura e seleção dos artigos, foi possível identifica-los conforme as classificações dos níveis de evidência. Os resultados são apresentados em quadros

Figura 1: Fluxograma do desenvolvimento da revisão integrativa

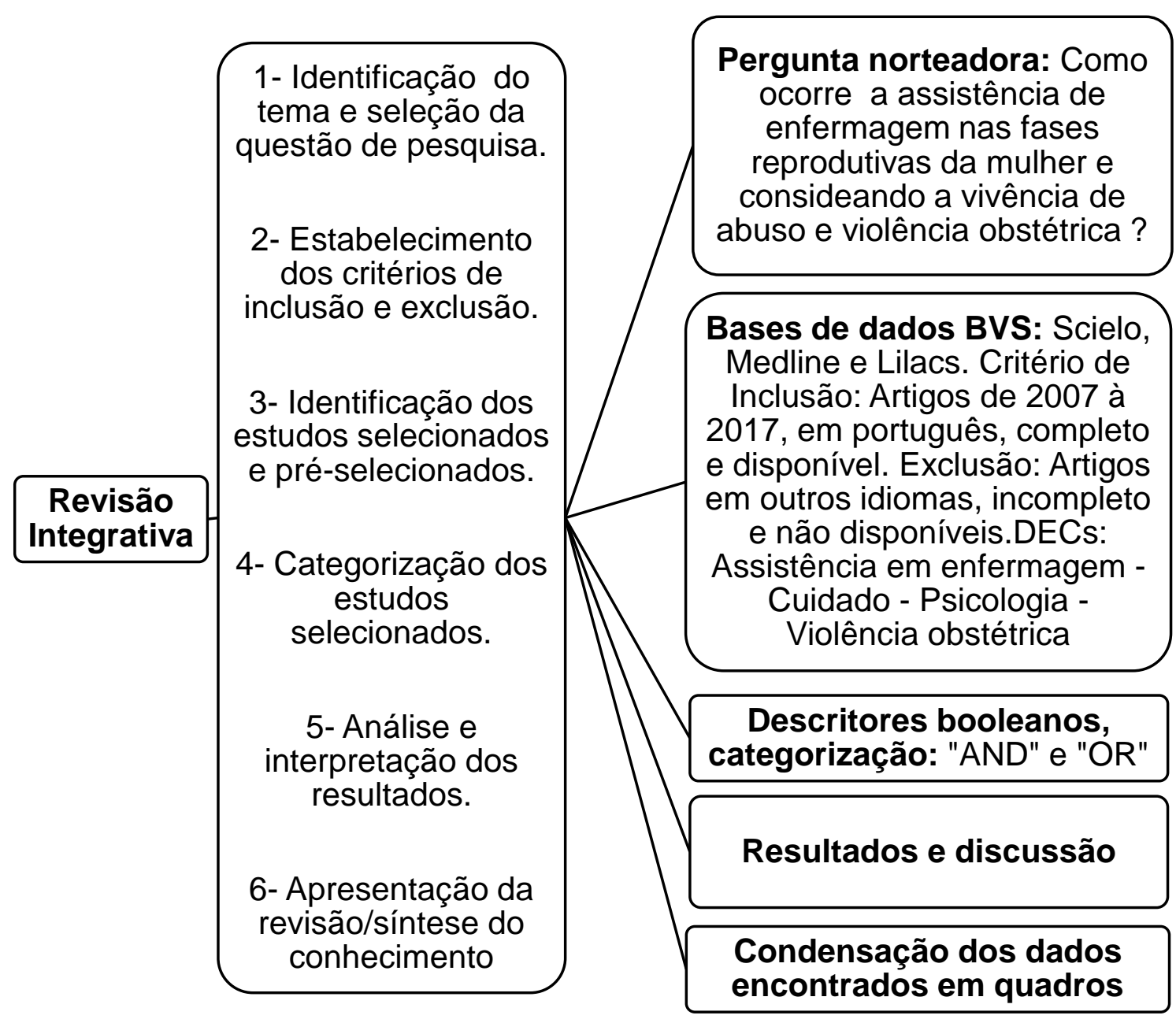




\section{Resultados e Discussão}

Mediante a identificação dos níveis de evidência das literaturas, destas, 9,09\% corresponderam a estudos de meta-análise - nível 1 e 90,90\%, se enquadraram no nível de evidência 4, que envolve tanto os estudos descritivos quanto os qualitativos. Com relação às regiões brasileiras que os artigos foram publicados, evidenciou-se que as revistas científicas se situam em três das cinco regiões do país, nordeste, sudeste e sul, apresentando respectivamente o percentual de artigos publicados em cada uma delas: 54,54\%, 36,36\% e 9,09\%.

De acordo com as literaturas estudadas, todos os materiais conceituam e detalham a VO vivenciada no PPP; algumas dessas agressões são: os toques vaginais frequentes e realizados por vários profissionais, episiotomia, proibição da ingesta líquida e alimentícia, restrição à posição horizontal, exclusão do protagonismo da mulher, negação da presença de um acompanhante, omissão e falta de informações fidedignas a respeito da evolução do trabalho de parto e situação atual do feto, entre outros (SANFELICE et al., 2014; LEISTER E RIESCO, 2013; SANTOS, MELO E CRUZ, 2015; PÉREZ, OLIVEIRA E LAGO, 2015; ALVARENGA E KALIL, 2016; OLIVEIRA et al, 2013).

Além desses artigos que abordam o tema central do estudo, foi possível identificar os pontos semelhantes entre os artigos e agrupar em três subgrupos temáticos, dos quais contribuem significativamente para a ocorrência da VO. Em destaque: 1- O alto índice de partos cesáreos (KNUPP, MELO E OLIVEIRA, 2008; ALVARENGA E KALIL, 2016), 2- A falta de humanização por parte dos profissionais (RATTNER, 2009; LEISTER E RIESCO, 2013; SANTOS, MELO E CRUZ, 2015; PÉREZ, OLIVEIRA E LAGO, 2015) e 3- Traumas que potencializam o desenvolvimento de transtornos psicológicos relacionados ao ciclo gravídicopuerperal (CANTILINO et al., 2010; LOBATO, MORAES E REICHENHEEIM, 2011).

No primeiro subgrupo temático, os artigos apontaram a grande demanda de partos cesáreos, tanto no serviço público quanto no privado; sendo este último o local que apresenta maior índice, cerca de $83 / 90 \%$, quando comparado ao serviço público essa taxa se encontra em 52\% dos partos (KNUPP, MELO E OLIVEIRA, 2008; ALVARENGA E KALIL, 2016). Em relação à idade das parturientes do estudo e o parto realizado, as mulheres que apresentaram idade igual ou maior que 35 anos, apresentaram um maior quantitativo de partos cesáreos. Para esse resultado, os autores apontaram como possível justificativa, a relação entre a idade avançada das mães como fator de risco para o aparecimento de patologias; enquanto as 
mulheres que estavam dentro da faixa etária entre 15-19 anos apresentaram maior realização dos partos por via vaginal (KUNPP, MELO E OLIVEIRA, 2008).

Enquanto síntese do segundo subgrupo temático, pôde-se evidenciar a ausência de "práticas humanizadas" por parte dos profissionais para com as gestantes; e a grosseria, a negligência, a dominação profissional por meio dos conhecimentos profissionais, a negação dos direitos maternos a respeito da escolha da via de parto, a falta de presença de acompanhante na hora do parto e informações fidedignas, foram os aspectos mais referenciados nos materiais (RATTNER, 2009; LEISTER E REISCO, 2015; SANTOS, MELO E CRUZ, 2015; PÉREZ, OLIVEIRA E LAGO, 2015).

Já no terceiro subgrupo temático, os artigos abordaram o surgimento de transtornos mentais que atingem as mulheres que vivenciaram na gestação, episódios traumáticos decorrentes da VO. Os sentimentos de tristeza, frustração, impotência e a presença dos pesadelos são fatores de riscos para que a mãe desenvolva a depressão pós-parto, a psicose pósparto e até mesmo retome a vida sexual (CANTILINO et al., 2010; LOBATO, MORAES E REICHENHEEIM, 2011).

A compilação e a análise dos resultados obtidos encontram-se expostas no Quadro 1, baseado em um instrumento validado contendo, o número da referência do artigo, ano de publicação, autores, título e objetivos. Já o segundo quadro evidencia a área da revista em que o artigo fora publicado, o tipo de pesquisa e o nível de evidência.

Quadro 1: Artigos selecionados para revisão integrativa de literatura

\begin{tabular}{|c|c|c|c|c|}
\hline ARTIGO & $\begin{array}{l}\text { ANO DE } \\
\text { PUBLICAÇÃO }\end{array}$ & AUTORES & TÍTULO & OBJETIVOS \\
\hline A1 & 2017 & $\begin{array}{l}\text { Tayse Ribeiro } \\
\text { de Oliveira, } \\
\text { Roxanny } \\
\text { Enolylara } \\
\text { Oliveira Lira } \\
\text { Costa, Nadiana } \\
\text { Lima Monte, } \\
\text { Juscélia Maria } \\
\text { de Moura } \\
\text { Feitosa Veras, } \\
\text { Maria Íris } \\
\text { Mendes da } \\
\text { Rocha Sá }\end{array}$ & $\begin{array}{l}\text { Percepção das } \\
\text { Mulheres sobre } \\
\text { violência obstétrica }\end{array}$ & $\begin{array}{l}\text { Caracterizar a violência obstétrica } \\
\text { vivenciada pelas mulheres durante o } \\
\text { processo parturitivo. }\end{array}$ \\
\hline $\mathrm{A} 2$ & 2016 & $\begin{array}{l}\text { Sarah Pereira } \\
\text { Alvarenga, }\end{array}$ & $\begin{array}{l}\text { Violência obstétrica: } \\
\text { como o mito "parirás }\end{array}$ & $\begin{array}{l}\text { Demonstrar que, apesar da violência } \\
\text { obstétrica ser extremamente comum } \\
\text { no Brasil, poucas mulheres sabem o }\end{array}$ \\
\hline
\end{tabular}


2014
José Helvécio
Kalil

Rafaella

Ayanne Alves

dos Santos,

Mônica Cecília

Pimentel de

Melo, Daniel

Dias Cruz

Bárbara

Angélica

Gómez Pérez,

Edilaine Varjão

Oliveira,

Mariana Santos

Lago

com dor" afeta a mulher Brasileira

Trajetória de humanização do parto no brasil a partir de uma revisão integrativa de literatura

Percepções de puérperas vítimas de violência institucional durante o trabalho de parto e parto

Do parto institucionalizado ao parto domiciliar

Sanfelice,

Fernanda de

Souza Freitas

Abbud,

Olívia

Separavich

Pregnolatto,

Michelle

Gonçalves da

Silva,

Antonieta Keiko

Kakuda Shimo

Nathalie Leister, Assistência ao parto: Maria Luiza

Gonzalez

Riesco história oral de mulheres que deram à luz nas décadas de 1940 a 1980

Ariane Thaise

Frello,

Telma Elisa

Carraro,

Mariely

Carmelina

Bernardi

Gustavo Lobato,

Cláudia L.

Moraes,

Michael E.
Cuidado e conforto no parto: estudos na Enfermagem brasileira

Magnitude da depressão pós-parto no Brasil: uma revisão sistemática que pode ser considerado uma violência e quais são os seus direitos.

Propõe a descrever a trajetória evolutiva do processo de parto no Brasil, com a finalidade de criar subsídios para o entendimento das atuais práticas de cuidado às gestantes e às parturientes nos dias atuais, enfatizando a proposta da humanização.

Analisar os resultados de pesquisas, sobre as percepções de puérperas vítimas de violência institucional, no seu trabalho de parto e parto, relacionando com poder e gênero.

Objetivou-se descrever a experiência vivenciada por um grupo de enfermeiras obstetras da cidade de Campinas, SP, Brasil, sobre o processo de transição do atendimento ao parto institucionalizado para o parto domiciliar, ocorrido no período de 2011 a 2013.

Compreender as transformações no modelo de assistência ao parto, a partir da experiência de mulheres que deram à luz no Estado de São Paulo, nas décadas de 1940 a 1980.

Identificar os estudos de Enfermagem que abordam os cuidados e conforto prestados no momento do parto, buscando

destacar lacunas existentes na área.

Realizar uma revisão dos estudos sobre a magnitude da depressão pósparto realizados no país até a presente data e apresentar as 
Reichenheim

A9

A10

2009

2008

\author{
Amaury \\ Cantilino, \\ Carla Fonseca \\ Zambaldi, \\ Everton Botelho \\ Sougey, \\ Joel Rennó Jr \\ Daphne Rattner
}

Humanização na
atenção a nascim e partos:

breve referencial

teórico

Virginia Maria

de Azevedo

Oliveira Knupp;

Enirtes Caetano

Prates Melo;

Rejane

Burlandi de

Oliveira

Transtornos psiquiátricos no pósparto

Distribuição do parto no município do Rio de Janeiro no período de 2001 a 2004 atenção a nascimentos

estimativas oriundas de cada estudo, discutir estes dados à luz das

diferenças metodológicas

e populacionais existentes entre eles.

Fazer uma atualização sobre os transtornos

psíquicos no pós-parto.

Movimentos pela luta da humanização da atenção a nascimentos e partos como uma resposta à mecanização na organização do trabalho profissional e à violência institucional, com crescente produção teórica. vaginal e da cesariana
Analisar a distribuição do parto vaginal e da cesariana no Município do Rio de Janeiro no período de 2001 a 2004

Fonte: Levantado pelos autores

Quadro 2:Classificação dos artigos conforme área de publicação da revista, tipo de estudo e nível de evidência.

\begin{tabular}{llll}
\hline ARTIGOS & $\begin{array}{l}\text { ÁREA DE } \\
\text { PUBLICAÇÃo }\end{array}$ & TIPO DE PESQUISA & NíVEL DE EVIDÊNCIA \\
\hline A1, A6 & Enfermagem & $\begin{array}{l}\text { Estudo descritivo com } \\
\text { abordagem qualitativa }\end{array}$ & Nível 4 \\
A2 & Interdisciplinar & Estudo qualitativo & Nível 4 \\
A3 & Multidisciplinar & $\begin{array}{l}\text { Estudo descritivo com } \\
\text { abordagem qualitativa }\end{array}$ & Nível 4 \\
A4, A7 A11 & Enfermagem & $\begin{array}{l}\text { Estudo descritivo } \\
\text { A5 }\end{array}$ & Meta-análise 4 \\
A8 & Enfermagem & Estudo descritivo & Nível 4 \\
A9 & Materno - Infantil & Estudo descritivo & Nível 4 \\
A10 & Interdisciplinar & Estudo descritivo & Nível 4 \\
\hline
\end{tabular}

Fonte: Levantado pelos autores 
Importa-nos destacar que a VO possui um caráter de normalidade por conta da sua prática rotineira, entretanto, não há um reconhecimento por parte das mulheres sobre essas ações com o verdadeiro conceito. As mulheres que se encontram no ciclo gravídico-puerperal desconhecem tais condutas como um tipo de VO (PÉREZ, OLIVEIRA E LAGO, 2015)

A ausência de informes colabora para uma luta enfraquecida pelos direitos da saúde reprodutiva das mulheres, contribui para a subnotificação e identificação desses atos.

Estudos mostram a incidência da VO entre as mulheres; cerca de " $25 \%$ das mulheres brasileiras já sofreram algum tipo de violência durante o seu trabalho de parto e parto"; já em outro estudo, foi evidenciado que uma entre quatro mulheres sofrem algum tipo de VO (VENTURY et al., 2010).

Porém, estima-se que devido à desinformação, esses números sejam ainda maiores. Com essa perspectiva e a imposição desse paradigma cultural referente à submissão feminina, torna-se difícil a desconstrução dessa ideologia. Entretanto, no momento que estas passam a conhecer que essa realidade pode ser diferente a qual se viveu, torna-se possível identificar as violências vividas (VENTURA, 2009).

Qualquer que seja o tipo da VO sofrida pode desencadear danos de baixo e grande impacto na vida da mulher, podendo também influenciar a relação com o meio familiar. A vivência das VOs pode ocorrer independente do seu conhecimento ou não, e podem gerar traumas e cicatrizes que podem ser aliviadas após o nascimento do seu filho ou que podem durar por uma vida toda (VENTURA, 2009; ALVARENGA E KALIL, 2016; OLIVEIRA, 2013).

Esses sentimentos negativos acarretados do PPP variam de mulher para mulher de acordo com a agressão sofrida e à sua intensidade, partindo dos profissionais que ferem o direito à autonomia nas decisões e com relação aos procedimentos que serão realizados em seu corpo, a privacidade e a informação (LEISTER E RIESCO, 2015).

A resposta do organismo e da mente dessas mulheres podem apresentar diversas sintomatologias, como "não conseguir mais ter relações sexuais com os seus parceiros, ter depressão pós-parto, pesadelos" (LEISTER E RIESCO, 2015; VENTURA, 2009; ALVARENGA E KALIL, 2016) e a psicose pós-parto (CANTILINO et al., 2010)

A ocorrência de um parto com eventos que geram traumas podem ser comparados com o transtorno do estresse pós-traumático (TEPT), a partir de sentimentos como coerção, desinformação, amedrontamento, inferioridade, tristeza, angustia, exclusão dos informes a 
respeito dos procedimentos e do seu estado de saúde, descuido por parte dos profissionais, perda do protagonismo; além disso, a realização dos procedimentos desnecessários considerados pelos profissionais e pelas gestantes como normais desencadeiam o TEPT (ROBINSON, 2007), como por exemplo, a episiotomia, cesariana sem indicação, falta de informação e negligência da equipe de saúde.

A fase gestacional causa ao organismo materno uma vulnerabilidade, e quando esta é vítima de agressões verbais, física, psicológica e infringem o seu direito de escolha, potencializam os riscos de desenvolver algum dano psicológico mais grave.

Com relação à depressão pós-parto, em um estudo bibliográfico foi possível indicar o período predisponente para o acometimento da depressão pós-parto, sendo ele estimado e variado no decorrer de um mês a seis meses (LOBATO, MORAES E REICHENHEEIM, 2011). A existência da PNHPN não configura a erradicação dos maus-tratos causados às mulheres em seu ciclo gravídico-puerperal; sabe-se que mesmo com a sua implantação ainda é notório a sua solidez. A modificação da conduta profissional é determinante para que de fato ocorra a melhoria objetivada pela política (SANTOS, MORAES E CRUZ, 2015).

É de extremamente necessidade a promoção um ambiente saudável, acolhedor, e que haja uma relação de confiança, de forma recíproca entre profissionais e parturientes, para que se possa diminuir o índice de sentimentos negativos e riscos que desenvolvem agravos psicológicos e traumas (CAMPOS, ALCÂNTARA E GOIS, 2015).

Dessa forma, cabe aos profissionais, principalmente os de enfermagem, que lidam por maior tempo e de forma mais próxima dos pacientes, e por se apresentarem em maior quantidade dentro dos serviços de saúde, uma práxis com ações humanizadas e sensíveis, de caráter individualizado, e que promovam conhecimentos. Permitindo assim, que as mulheres reconheçam seus direitos, que tenham o respeito por suas decisões e valorização pelo seu protagonismo (FRELLO, CARRARO E BERNARDI, 2011).

O desejo de um parto mais naturalizado e sem intervenções desnecessárias não deve povoar apenas o imaginário materno, mas, deve ser realizado rotineiramente nos serviços de assistência à mulher gestante, como está preconizado pela PNHPN, que viabiliza a autonomia e o protagonismo da mulher. 


\section{Considerações Finais}

Com este estudo, pôde-se concluir que as condutas do profissional enfermeiro e de qualquer outro profissional da área da saúde que lidem com mulheres do PPP, deveriam estar pautadas PNHPN, que objetiva promover uma assistência de qualidade e integral desde o prénatal até o puerpério e ao recém-nascido, valorizando sua a autonomia e protagonismo, entretanto, as práticas referendam alarmante crescimento de cesarianas e outras VOs, muitas destas, desconhecidas pelas mulheres e ampla sociedade.

É imprescindível que haja o repasse de qualquer informação sobre a saúde da mãe e do seu bebe quanto aos procedimentos que serão realizados, valorizando a escolha materna; pois assim, contribuirá para a redução dos níveis de estresse, insegurança e ansiedade, aumentando a confiança profissional-paciente e diminuição dos traumas psicológicos e agravos a saúde relacionados a essas vivências negativas, que interferem no seu bem estar e do modo como afetará a sua vida, podendo não conseguir mais ter relações sexuais com o seu (ua) parceiro (a), apresentar risco para a depressão pós-parto ou até mesmo ser acometida, pesadelos, psicose pós-parto, baixa autoestima, tristeza e impotência, que podem durar dias, meses ou até mesmo não serem superados.

Cabe aos profissionais, colocar em prática os princípios da PNHPN e tratar o ser humano com toda a sua integralidade, direitos e especificidade.

Com o avançar dos anos, a observância é de que a VO tem se tornado comum entre as mulheres e considerada uma prática rotineira entre alguns profissionais de saúde, devido a sua tamanha frequência. Entretanto, não há um total reconhecimento dessas práticas agressivas, negativas e prejudiciais para o binômio mãe-filho por parte do público feminino; é identificado por algumas mulheres que são atos errôneos, porém o seu real conceito não é conhecido, o que dificulta a luta e o posicionamento das mesmas pelos seus direitos. Dessa forma, idealiza-se que esse estudo contribua para o entendimento, o estímulo para reivindicação dos direitos e acompanhamento das lutas pelo protagonismo das mulheres e usuárias dos serviços específicos; assim como, para conscientizar os acadêmicos e profissionais da área da saúde para o quão danoso é reproduzir esses atos. 


\section{Referências}

ALVARENGA, S. P., KALIL, J. H. VIOLÊNCIA OBSTÉTRICA: como o mito "parirás com dor" afeta a mulher brasileira. Revista da Universidade Vale do Rio Verde, Três Corações, v. 14, n. 2, p. 641-49, Ago./dez, 2016

AZEVEDO, Júlio Carvalho. Precisamos falar sobre a violência obstétrica. 2015. Disponível em: <http://www.conjur.com.br/2015-mai-16/julio-azevedo-precisamos-falar-violencia-obstetrica>. Acesso em: 03 de abril de 2017.

BRASIL. Ministério da 2015. <http://portalsaude.saude.gov.br/index.php/cidadao/principal/agencia-saude/27782-pela-primeira-veznumero-de-cesarianas-nao-cresce-no-pais> Acesso em 13 de março de 17.

BRASIL. Ministério da saúde secretaria de atenção à saúde. Portaria $N^{o}$ 306, de 28 de março de 2016. Aprova as Diretrizes de Atenção à Gestante: a operação cesariana.

BRASIL. Agência Nacional a Saúde Suplementar. Mapa assistencial a saúde suplementar, Abril, 2013.

BRASIL. Humanização do parto e do nascimento. Ministério da Saúde. Universidade Estadual do Ceará. - Brasília: Ministério da Saúde, (Cadernos HumanizaSUS), v. 4, 2014.

BREVIDELLI, M. M., SERTÓRIO, S. C. TCC: trabalho de conclusão de curso: guia prático para docentes e alunos da área da saúde. 4a ed. São Paulo: Iátria; 2010. Pag 10.

CAMPOS, M. E. A. L., ALCANTARA, D. F., GOIS, L. P. B. L. Incidência e características de cesáreas e partos normais: um estudo comparativo. Congresso Brasileiro dos Conselhos de Enfermagem (CBCEnf), João Pessoa-PB, 2015.

CANTILINO, A. et al. Transtornos psiquiátricos no pós-parto. REV PSIQ CLÍN.São Paulo, 37(6):27884, 2010.

FRELlO, A. T., CARRARO, T. E., BERNARDI, M. C. Cuidado e conforto no parto: estudos na Enfermagem brasileira. Revista Baiana de Enfermagem, Salvador, v. 25, n. 2, p. 173-84, maio/ago. 2011.

GACETA OFICIAL DE LA REPÚBLICA BOLIVARIANA DE VENEZUELA. Nº40.551, Ano CXLII - Mês II, Nº40.551, 28 de novembro de 2014. Caracas.

GRUPO ANIMA EDUCAÇÃO. Manual Revisão Bibliográfica Sistemática Integrativa: a pesquisa baseada em evidências. Grupo Ănima Educação. Belo Horizonte: Copyright; 2014. Pag 11.

KNUPP, V. M. A. O., MELO, E. C. P., OLIVEIRA, R. B. Distribuição do parto vaginal e da cesariana no município do rio de janeiro no período de 2001 a 2004. Esc Anna Nery Rev Enferm, Rio de Janeiro, 12 (1): 39-44, março, 2008.

LEISTER, N., RIESCO, M. L. G. Assistência ao parto: história oral de mulheres que deram à luz nas décadas de 1940 a 1980. Texto Contexto Enferm, Florianópolis, 22(1): 166-74, Jan-Mar, 2013. 
LOBATO, G., MORAES, C. L., REICHENHEEIM, M. E. Magnitude da depressão pós-parto no Brasil: uma revisão sistemática. Rev. Bras. Saúde Matern. Infant., Recife.11 (4): 369-79, out./dez, 2011.

PARTO DO PRINCÍPIO-MULHERES EM REDE PELA MATERNIDADE ATIVA DOSSIÊ DA VIOLÊNCIA OBSTÉTRICA. "Parirás com dor". Elaborado para a CPMI Violência Contra as Mulheres. 2012.

PÉREZ, B. A. G., OLIVEIRA, E. V., LAGO, M. S. Percepções de puérperas vítimas de violência institucional durante o trabalho de parto e parto. Revista Enfermagem Contemporânea, Bahia, 4(1):6677, Jan./Jun, 2015.

RATTNER, D. Humanização na atenção a nascimentos e partos: ponderações sobre políticas públicas. Interface. Botucatu, 13, 595-02, 2009.

ROBINSON, V. Transtorno de estresse pós-traumático. AIMS Journal, Arizona-EUA, v.19, n.1. 2007.

SANFELICE, C. F. O. et al. Do parto institucionalizado ao parto domiciliar. Rev Rene. Fortaleza, 15, 362-70. mar-abr/2014.

SANTOS, R. A. A., MELO, M. C. P., CRUZ, D. D. Trajetória de humanização do parto no brasil a partir de uma revisão integrativa de literatura. Caderno de Cultura e Ciência, Universidade Regional do Cariri-URCA. v.13, n.2, 76-89, Março, 2015.

SOUZA, M. T., SILVA, M. D., CARVALHO, R. Revisão integrativa: o que é e como fazer. Einstein. 2010;Pag 102-06.

VENTURA, M. Direitos Reprodutivos no Brasil. Fundo de População das Nações Unidas - UNFPA, Brasília, 3a ed., 2009.

VENTURY, G., BOKANY, V., DIAS, R., ALBA, D., ROSAS, W., FIGUEIREDO, N. Mulheres brasileiras e gênero nos espaços públicos e privado. Pesquisa de opinião pública. Fundação Perseu Abramo e SESC. 2010.

\section{Como citar este artigo (Formato ABNT):}

SILVA, Fabiana Laranjeira; SOUZA, Ana Lívia Siqueira; LEITE-SALGUEIRO, Claudia Daniele Barros; BARBOSA, Luciclaudio da Silva; LOBATO, Luzineide; PEREIRA, Juliane da Silva. O Protagonismo do Enfermeiro na Política Nacional de Humanização do Parto e do Nascimento e com vistas a Erradicação de Abuso e Violência Obstétrica. Id on Line Rev.Mult. Psic., 2018, vol.12, n.41, p.1068-1082. ISSN: 1981-1179.

Recebido: 11/07/2018.

Aceito: $26 / 07 / 2018$ 